

\title{
DESEMPEÑO TÉRMICO DE FACHADAS VERDES TRADICIONALES DE ORIENTACIÓN ESTE EN VIVIENDAS SERIADAS EMPLAZADAS EN CLIMAS ÁRIDOS
}

\author{
THERMAL PERFORMANCE OF TRADITIONAL EAST FACING GREEN \\ FACADES IN TRACT HOUSING LOCATED IN ARID CLIMATES
}

\author{
PABLO ABEL SUAREZ \\ Arquitecto, Becario Doctora \\ Instituto de Ambiente, Hábitat y Energía (INAHE - CCT CONICET), \\ Consejo Nacional de Investigaciones Científicas y Técnicas \\ (CONICET) Mendoza, Argentina \\ https://orcid.org/0000-0002-0032-3278 \\ suarezpablo91@gmail.com
}

\author{
MARÍA ALICIA CANTÓN \\ Diplomada en Estudios Avanzados en Arquitectura \\ Investigadora principal Instituto de Ambiente, Hábitat y Energía \\ (INAHE - CCT CONICET), Consejo Nacional de Investigaciones \\ Científicas y Técnicas (CONICET) Mendoza, Argentina \\ https://orcid.org/0000-0002-8714-9697 \\ macanton@mendoza-conicet.gob.ar
}

ÉRICA CORREA

Doctora en Ciencias, Investigadora Independiente Independiente, Instituto de Ambiente, Hábitat y Energía (INAHE CCT CONICET), Consejo Nacional de Investigaciones Científicas y Técnicas (CONICET) Mendoza, Argentina https://orcid.org/0000-0003-1690-076X ecorrea@mendoza-conicet.gob.ar

\section{RESUMEN}

La infraestructura verde constituye una estrategia de mitigación de las temperaturas urbanas y edilicias. El presente trabajo evalúa el impacto de un tipo de Sistema de Enverdecimiento Vertical (SEV), las Fachadas Verdes Tradicionales (FVT), en la condición térmica de viviendas localizadas en el Área Metropolitana de Mendoza, Argentina; cuyo clima es seco desértico (BWk - Köppen-Geiger). Con tal fin, se monitorearon, durante dos veranos consecutivos, dos casos de estudio: una vivienda con FVT, en orientación este, y una vivienda testigo de igual tipología y materialidad. Se registraron datos de temperatura ambiente exterior e interior; superficial exterior e interior y radiación horizontal. Se hallaron disminuciones de hasta $3.1^{\circ} \mathrm{C}$ en la temperatura ambiente interior de las viviendas con

$\mathrm{FVT}$, de hasta $27.4^{\circ} \mathrm{C}$ en muros exteriores y de $6.5^{\circ} \mathrm{C}$ en muros interiores. Las magnitudes de los resultados encontrados demuestran el potencial de la aplicación de esta estrategia en un clima árido.

Palabras clave

zonas áridas, arquitectura bioclimática, viviendas unifamiliares, Sistemas de Enverdecimiento Vertical.

\section{ABSTRACT}

Green infrastructure is a strategy for mitigating urban and building temperatures. This work assesses the impact of a type of Vertical Greenery System (VGS), the Traditional Green Façades (TGF), on the thermal condition of dwellings located in the Metropolitan Area of Mendoza, Argentina, whose climate is dry desert (BWk - Köppen-Geiger). To this end, two case studies were monitored for two consecutive

summers: a dwelling with an east-facing TGF and a control dwelling of the same typology and materiality. Outdoor and indoor ambient temperature data were recorded: surface exterior and interior, and horizontal radiation. Decreases of up to $3.1^{\circ} \mathrm{C}$ in the indoor ambient temperature of FVT dwellings, of up to $27.4^{\circ} \mathrm{C}$ on exterior walls and $6.5^{\circ} \mathrm{C}$ on interior walls were found. The magnitudes of the results found show the potential of applying this strategy in an arid climate.

\section{Keywords}




\section{INTRODUCCIÓN}

En áreas densamente pobladas existen impactos negativos propios del avance de la urbanidad sobre el medio ambiente, desde emisiones de carbono y el incremento de las temperaturas medias del aire, hasta la depredación de zonas productivas periféricas y la destrucción de los ecosistemas. De acuerdo a datos de la Agencia Internacional de Energía (OCED / IEA, 2017), las ciudades ocupan el $3 \%$ de la superficie del planeta y, además de provocar el aumento de las temperaturas medias del aire, son responsables del $67 \%$ del consumo energético global. A su vez, el Programa de Naciones Unidas para el Medio Ambiente (PNUMA) indica que el $75 \%$ de la infraestructura que existirá en el 2030, aún no fue construida. Esto representa una oportunidad para crear "ciudades limpias y verdes", eficientes y resilientes. Una estrategia para alcanzar este objetivo es el enverdecimiento urbano.

La infraestructura verde genera beneficios energéticoambientales: a escala urbana, disminuye la isla de calor y aumenta el confort de los espacios públicos $y$, a escala edilicia, reduce el consumo de energía para acondicionamiento de los espacios interiores. Investigaciones recientes han determinado que en un clima templado el incremento de la infraestructura verde en un $10 \%$ podría reducir las temperaturas medias del aire urbano $2.5^{\circ} \mathrm{C}$ (Gill, Handley, Ennos y Pauleit, 2007) y que en un clima seco árido se puede disminuir $3.8^{\circ} \mathrm{C}$ la temperatura ambiente en un lugar arbolado (Salas y Herrera, 2017). Además, los espacios verdes generan beneficios para la salud y bienestar de los ciudadanos (Contesse, Van Vliet y Lenhart, 2018). Dado que el fenómeno de consolidación de las ciudades ha limitado el potencial de incorporación de estructuras verdes tradicionales -parques, plazas, arbolado de alineación-, han surgido nuevas tipologías de vegetación asociadas a cubiertas y muros verdes.

El desarrollo de conocimiento vinculado a los Sistemas de Enverdecimiento Vertical (SEV) ha crecido en función de su relevancia a nivel mundial en la última década (Bustami, Belusko, Ward y Beecham, 2018). Muestran probada eficiencia en la disminución de las temperaturas de los espacios habitables y sus consecuentes impactos en los consumos de energía. Los resultados varían en su magnitud según el tipo de clima donde se aplican los SEV, registrando máximas disminuciones de temperatura superficial exterior del orden de los $34^{\circ} \mathrm{C}$ (Suklje, Saso y Arkar, 2016) en climas del tipo $\mathrm{Cfa} / \mathrm{Cfb}$ (templado cálidos húmedos, verano caliente), de temperatura ambiente interior del orden de los $5^{\circ} \mathrm{C}$ (Haggag, Hassan y Elmasry, 2014) en climas del tipo BWh (árido desértico, verano caliente) y de temperatura ambiente exterior del orden de los $3.3^{\circ} \mathrm{C}$ (Wong, Kwang Tan, Tan, Chiang y Wong, 2010) en climas del tipo Af (Ecuatorial húmedo).
Mayoritariamente, los estudios se han realizado en países europeos, asiáticos y norteamericanos; en climas del tipo templado cálido, tanto húmedo como seco -Csa, Cfa/Cfb-. Los resultados han mostrado similares o mejores rendimientos para los SEV, en cuanto a las reducciones de temperatura, en climas áridos respecto a climas húmedos.

El Área Metropolitana de Mendoza, Argentina (AMM), presenta un clima árido desértico (BWk - KöppenGeiger). Desde el punto de vista de la presencia de espacios vegetados, cuenta en su estructura con una importante cantidad de espacios abiertos forestados. Sin embargo, el proceso de crecimiento y densificación urbana no ha ido acompañado por un proceso de densificación del verde urbano y, además, ha limitado la disponibilidad de vacíos urbanos que permiten la incorporación de espacios verdes tradicionales. En consecuencia, incrementar las áreas verdes implica implementar nuevas tecnologías de vegetación como lo son, entre otras, los SEV.

Los beneficios termo-energéticos de los SEV están asociados a diversos efectos. Primero, al efecto de sombreo, que ubica a los SEV como interceptores de la radiación solar incidente, directa o indirecta. Este efecto, dependiendo de la orientación del muro, es importante en climas con fuerte incidencia solar (Othman y Sahidin, 2016). En segundo lugar, al efecto de enfriamiento que reduce la temperatura del aire y aumenta la humedad liberando vapor de agua de las plantas a la atmósfera (Wong y Baldwin, 2016). Está comprobado que el alcance de este se extiende hasta $60 \mathrm{~cm}$ de su superficie (Wong et al., 2010). También hay que mencionar el efecto de aislamiento, producido por los estratos que componen el paquete constructivo de los SEV que interfieren en la transmisión de calor de las envolventes edilicias. Y, finalmente, el efecto barrera contra el viento, que provoca disminuciones en pérdidas y ganancias de calor por convección debido a la rugosidad del follaje que obstaculiza la circulación de aire. Respecto al impacto del SEV en el ahorro de energía, la literatura internacional registra valores del orden de 58.9\% (Coma et al., 2017). En cuanto a los beneficios ambientales, se registraron valores de absorción de emisiones de carbono que oscilan entre 0.14 y $0.99 \mathrm{Kg} / \mathrm{m}^{3}$ (Marchi, Pulselli R., Marchettini, Pulselli F. y Bastianoni, 2015). Ello, además del impacto que genera en el grado de absorción acústica y el aporte a la preservación de la biodiversidad. Por último, las fachadas vegetadas aumentan la percepción de confort, relajación y mejoran el estado de ánimo (Elsadek, Liu y Lian, 2019).

Los SEV se agrupan en dos categorías: Pared Viva (PV) y Fachadas Verdes (FV) (Figura 1). Las FV son aquellos sistemas en los que hay plantas trepadoras y/o arbustos colgantes cubriendo un área determinada. Pueden dividirse en tres tipologías: Fachadas Verdes 


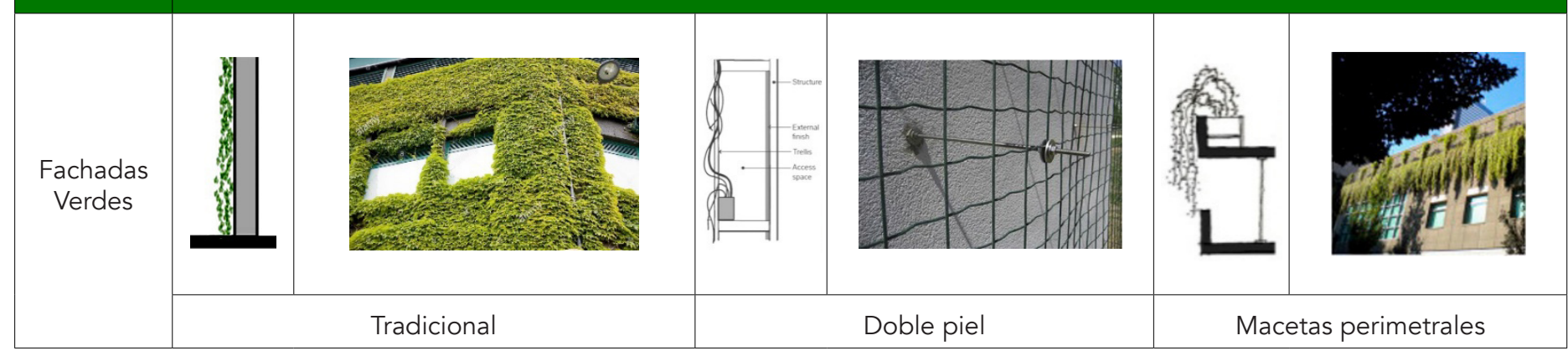

Figura 1. Tipologías de FV. Fuente: Elaboración de los autores.

Tradicionales (FVT), donde la planta se adosa al muro del edificio; Fachadas Verdes tipo Doble Piel (FVDP) o cortina verde, donde existe una estructura suplementaria que se separa una distancia variable de la pared del edificio; y Fachadas Verdes con Macetas Perimetrales (FVMP), donde pueden alojarse macetas con plantas trepadoras y/o arbustos colgantes para generar una cortina verde.

Las FVT son sistemas de aplicación simple, de bajo costo y reducido impacto en la construcción existente. En el caso de Mendoza, esta estrategia es de aplicación espontánea y ampliamente difundida en las áreas residenciales de baja densidad. Los beneficios se clasifican en dos categorías: termo-energéticos y ambientales.

Los beneficios termo-energéticos han sido analizados extensamente en la literatura internacional. Sin embargo, es acotado el desarrollo de conocimiento en relación con la incidencia de las FVT en el comportamiento térmico de los espacios interiores en climas del tipo desértico, como también respecto al análisis del impacto diferencial del uso de la estrategia, de acuerdo con la orientación de la fachada.

En ese contexto, Alexandri y Jones (2008) determinan que la radiación máxima recibida en verano en los planos verticales orientados al este y al oeste es 1.65 veces mayor a la recibida en los planos Norte y Sur, para el caso de Atenas, Grecia, cuya latitud $\left(32^{\circ} \mathrm{N}\right)$ es equiparable con la de la ciudad de Mendoza (37\% S). Susorova, Angulo, Bahrami y Stephens (2013), por su parte, miden el efecto de las FVT sobre muros de edificios en las cuatro orientaciones en Chicago, hallando magnitudes mayores, del orden de 4 y 5 veces más, para las orientaciones este y oeste. Coma et al. (2017) determinan que las fachadas tipo FVDP son más efectivas en orientaciones oeste y este que en orientación norte. Estudios recientes atribuyen mayores magnitudes de enfriamiento y ahorro de energía producida por una FV en orientaciones este y oeste en condiciones de verano (Pérez, Coma, Sol y Cabeza, 2017) (Kontoleon y Eumorfopoulou, 2010). De lo descripto, se desprende que los SEV generan los mayores impactos en orientaciones este y oeste cuyos planos verticales son los más demandados por la radiación solar.

En consecuencia, el presente trabajo tiene por objetivo evaluar el impacto de las FVT con orientación este, sobre las temperaturas superficiales, interiores y exteriores, y la condición térmica de los espacios interiores en viviendas unifamiliares seriadas del AMM, Argentina.

\section{HIPÓTESIS DE TRABAJO}

En climas áridos, las nuevas tecnologías de vegetación, en particular las Fachadas Verdes Tradicionales, constituyen una estrategia de acondicionamiento bioclimático pasivo debido a su capacidad de atenuar las temperaturas superficiales de la envolvente edilicia y aproximar a las condiciones de confort a los espacios habitables en la estación cálida.

\section{METODOLOGÍA}

\section{CASOS DE ESTUDIO}

Para el desarrollo de este estudio se seleccionó dos viviendas localizadas en un barrio de tipología seriada en Guaymallén, Mendoza. Ambas son del tipo morfológico de casa compacta, ampliada, estructurada en dos niveles. La Figura 2 muestra la localización de las viviendas en el contexto del Área Metropolitana de Mendoza (AMM). Y, en la Figura 3, se observa el tipo morfológico de los edificios junto al estudio de asoleamiento hora a hora de la radiación solar incidente sobre fachada este. 

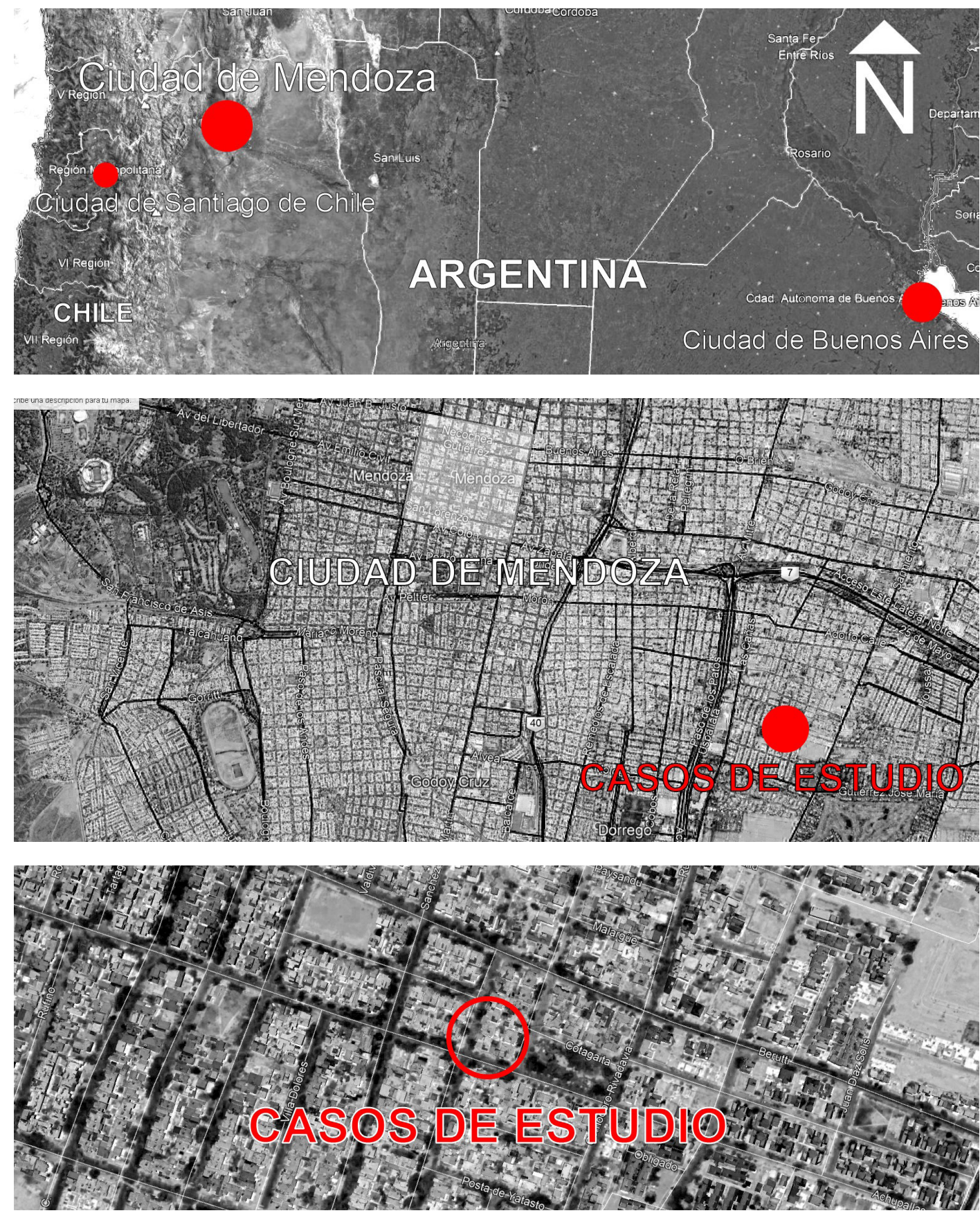

Figura 2. Localización de las viviendas en el contexto de AMM. Fuente: Elaboración de los autores.

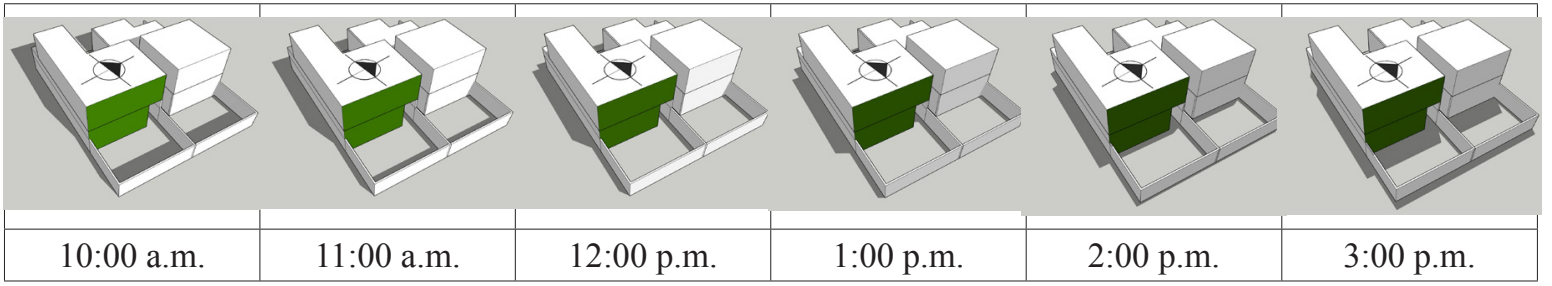

Figura 3. Estudio de asoleamiento hora a hora de radiación solar incidente sobre fachada este. Fuente: Elaboración de los autores. 


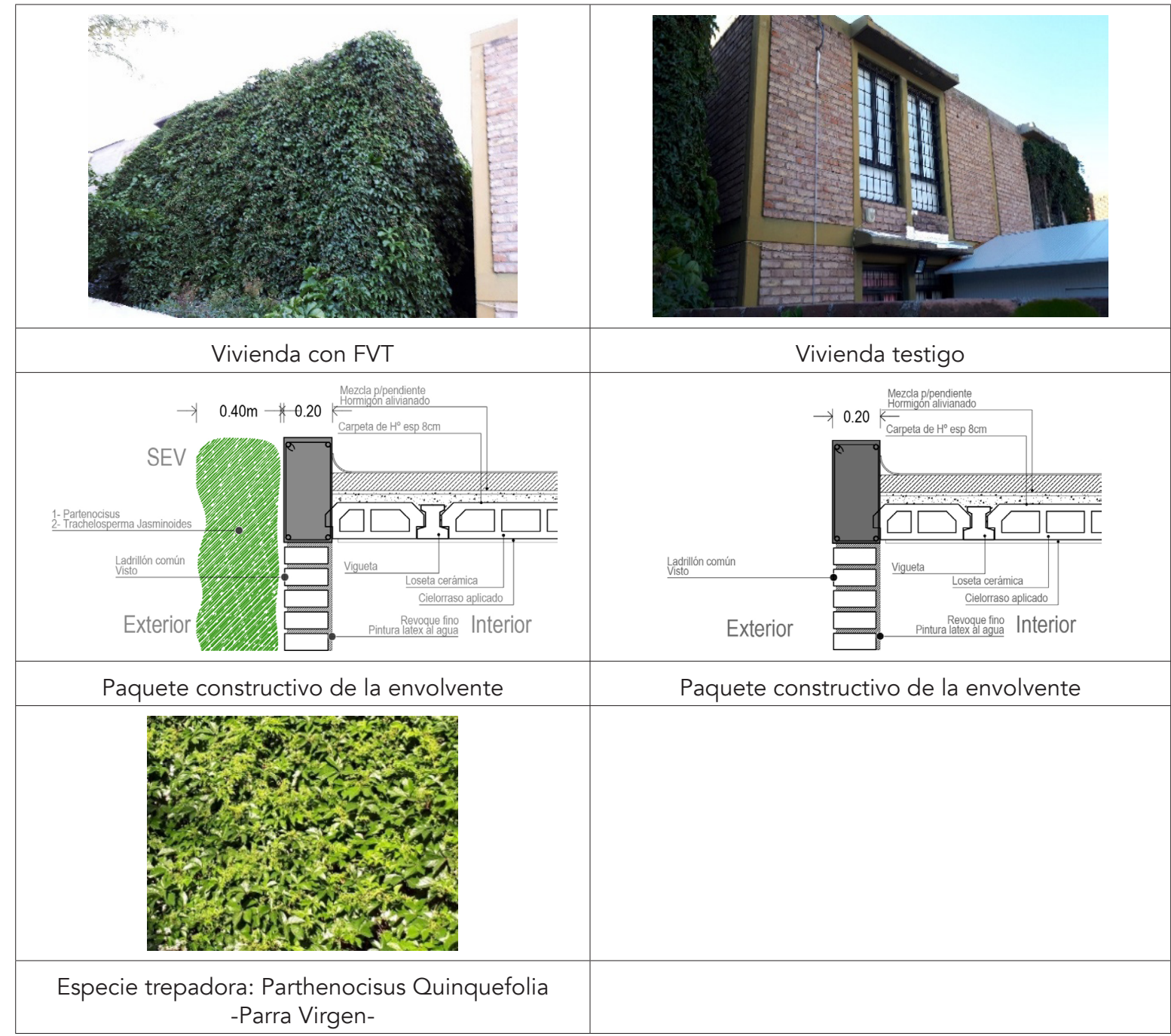

Figura 4. Casos de estudio. Fuente: Elaboración de los autores.

Tecnológicamente, los casos de análisis evidencian el tipo constructivo característico de áreas sísmicas: estructura de hormigón armado y mampostería de ladrillo visto. Las cubiertas son planas, conformadas por losas de hormigón alivianadas, aisladas térmica e hidráulicamente. Una de ellas presenta el sistema de FVT en la fachada interna de la vivienda orientada al este y en la fachada lateral orientada al norte. La FVT es un sistema simple conformado por una especie de planta trepadora del género Parthenocissus Quinquefolia, de la familia Vitaceae, localmente denominada Parra Virgen -especie originaria de América del Norte y Central; del tipo leñosa, caduca, resistente, crecimiento enérgico, alta densidad foliar y adaptabilidad (tolera la mayoría de los suelos y condiciones climáticas), baja permeabilidad solar y mantenimiento, ampliamente utilizada en la región-. La misma se desarrolla adosada al muro con un espesor promedio de $40 \mathrm{~cm}$ en toda su extensión y cubre el $100 \%$ del muro de mampostería que conforma la fachada este de la vivienda (Figura 4).

\section{MONITOREO DE CASOS DE ESTUDIO}

Para la evaluación de la incidencia de la FVT en el comportamiento térmico de las fachadas de orientación este de las viviendas, las variables medidas fueron: temperatura y humedad del aire exterior en los espacios abiertos públicos y privados colindantes con la vivienda, temperatura y humedad del aire interior y temperatura superficial interior y exterior en muros. Los sensores utilizados para medir la temperatura y humedad relativa ambiente fueron del tipo termistor y termocupla - $\mathrm{HOBO}$ Onset, UX100-003, UX120-. La localización de los sensores y el equipamiento utilizado se presentan en la Figura 5.

La toma de datos se registró en dos períodos: verano 2019 -21/01 al 05/02- y 2020 -30/01 al 14/02-, durante 15 días, mediante el registro de datos llevado a cabo cada 15 minutos. Los sensores fueron calibrados previamente para garantizar la confiabilidad de los datos obtenidos. 


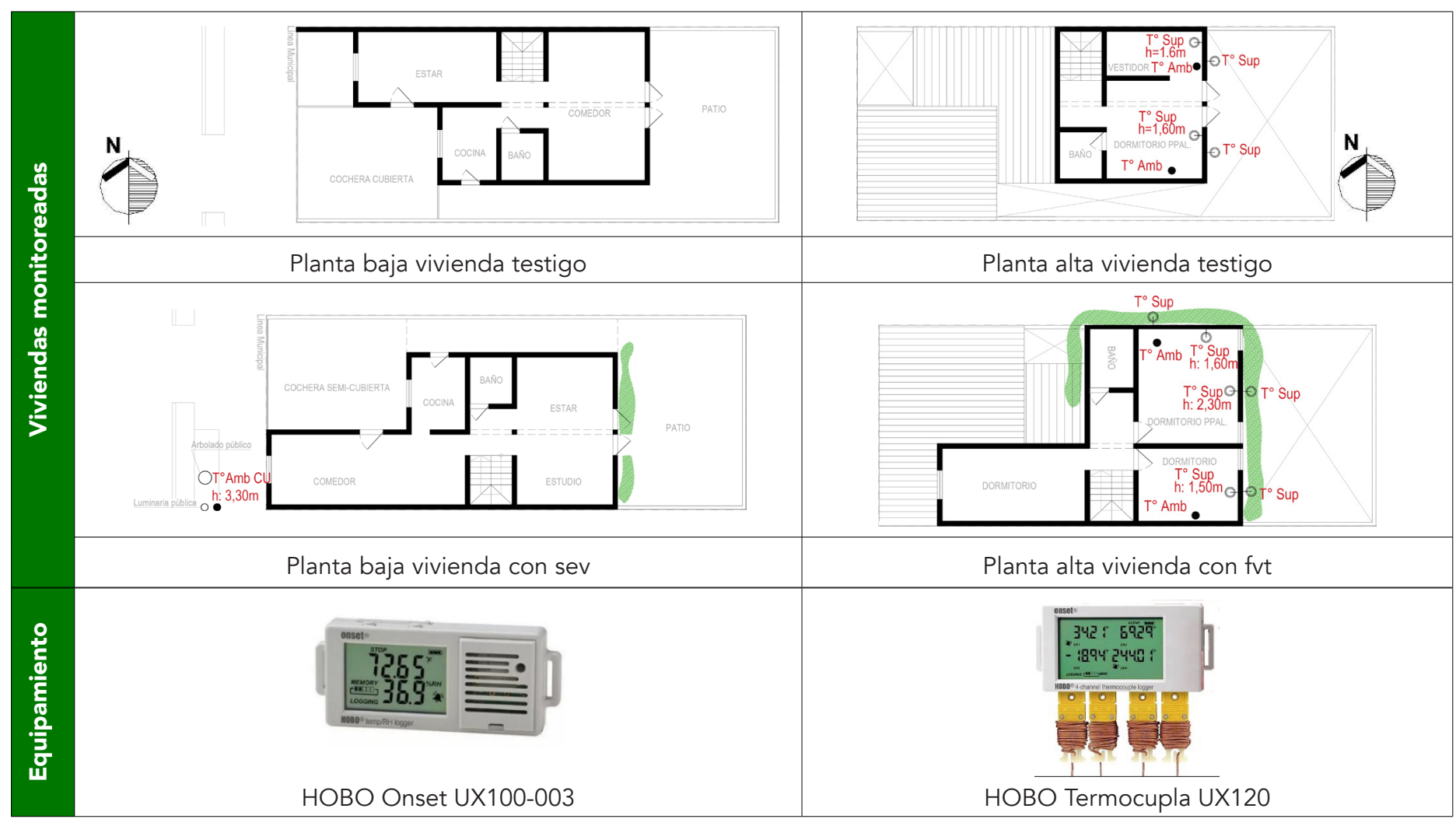

Figura 5. Casos de estudio: ubicación de sensores y equipamiento utilizado. Fuente: Elaboración de los autores

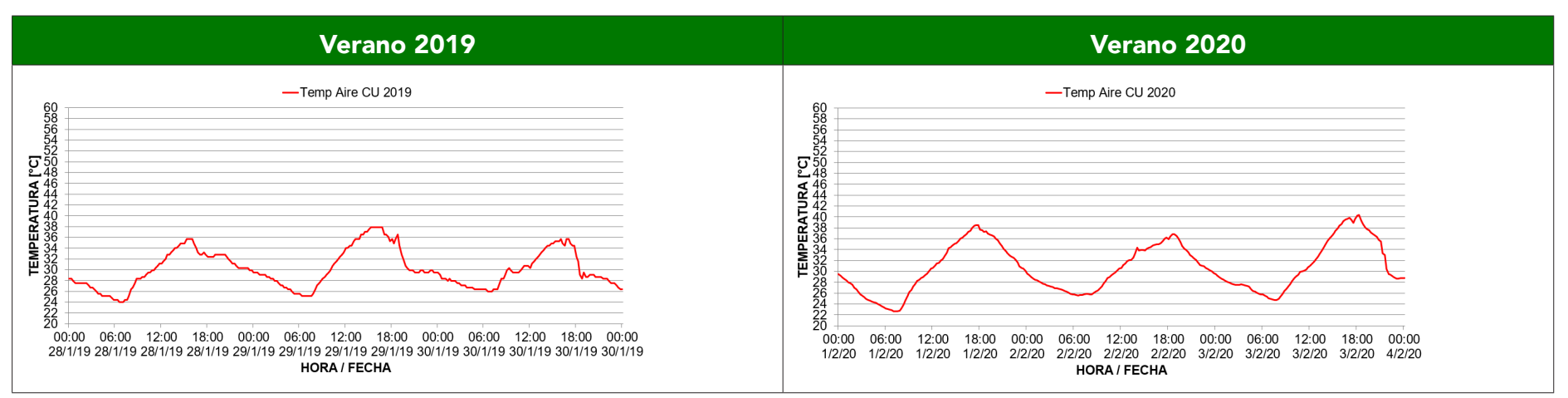

Figura 6. Temperatura del aire en el cañón urbano para los días seleccionados. Fuente: Elaboración de los autores.

\section{SELECCIÓN DE DÍAS DE ANÁLISIS}

A efecto de analizar comparativamente la respuesta térmica de los casos de estudio evaluados con y sin FVT, se eligieron aquellos días cuyas características fueran representativas de un día de verano propio del microclima local, dentro del período de 30 días en que se registraron las mediciones. En función de ello, los criterios de selección fueron: temperaturas máximas y mínimas más elevadas, alta heliofanía y baja velocidad de viento. Los días seleccionados en cada período fueron: del 28 al 30 enero de 2019, y del 1 al 3 de febrero de 2020. La Figura 6 expone el comportamiento de la temperatura del aire en el cañón urbano para los días de análisis. La forma de la curva refleja que los días analizados presentan un comportamiento semejante tanto en cuanto a la curtosis de la curva de temperatura del aire exterior como a la amplitud de esta. Se verifica que los picos de máxima y mínima temperatura se corresponden en magnitud y ocurrencia horaria a lo largo del tiempo.

Considerando el comportamiento de las curvas y del análisis cuantitativo de los datos para el período 2019, se observan rangos de temperaturas diarias que varían desde $35.7^{\circ} \mathrm{C}$ a $37.9^{\circ} \mathrm{C}$, para las máximas, y entre $24.0^{\circ} \mathrm{C}$ y $26.0^{\circ} \mathrm{C}$, para las mínimas. En los días correspondientes al verano 2020, se registran valores de temperaturas diarias que oscilan entre los $36.8^{\circ} \mathrm{C}$ y los $40.4^{\circ} \mathrm{C}$, para las máximas, y entre los $22.7^{\circ} \mathrm{C}$ y los $25.6^{\circ} \mathrm{C}$, para las mínimas (Tabla 1). 


\begin{tabular}{|l|l|l|l|l|l|l|}
\hline & Temp. máx. & Temp. mín. & $\begin{array}{c}\text { Temp. } \\
\text { prom. total. }\end{array}$ & $\begin{array}{c}\text { Temp. } \\
\text { prom. mín. }\end{array}$ & $\begin{array}{c}\text { Temp. } \\
\text { prom. máx. }\end{array}$ & $\begin{array}{c}\text { Amplitud } \\
\text { prom. }\end{array}$ \\
\hline $\begin{array}{l}\text { Período 2019: 28/01 - } \\
\text { 30/01 }\end{array}$ & 37.9 & 24.0 & 30.4 & 25.0 & 36.4 & 11.4 \\
\hline $\begin{array}{l}\text { Período 2020: 01/02 - } \\
\text { 03/02 }\end{array}$ & 404 & 22.7 & 30.9 & 24.3 & 38.6 & 14.2 \\
\hline
\end{tabular}

Tabla 1. Temperaturas máximas, medias y mínimas promedio para los períodos analizados. Fuente: Elaboración de los autores.

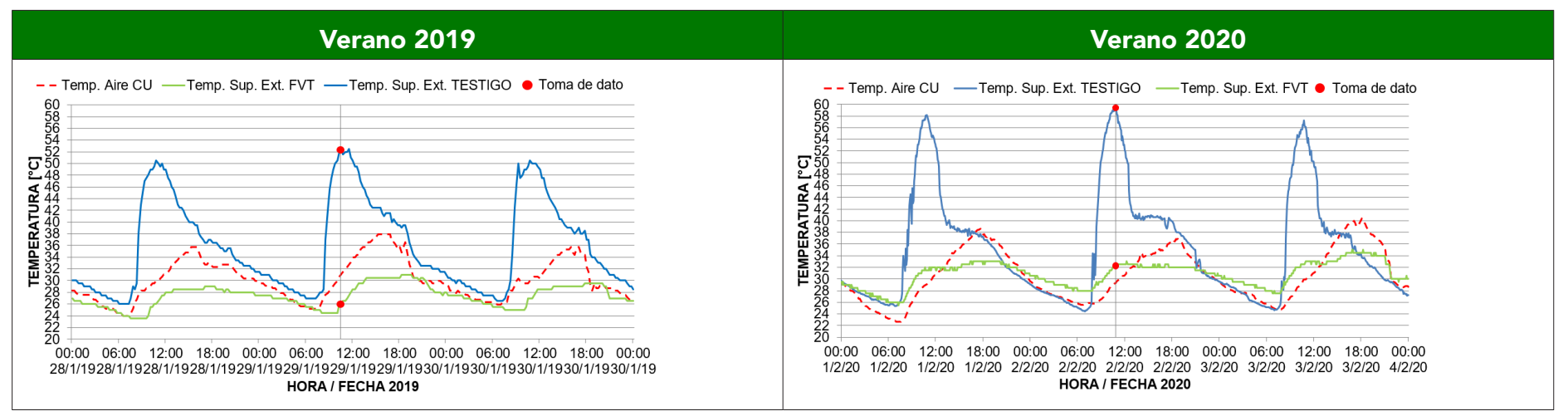

Figura 7. Temperatura superficial exterior: caso testigo y caso con FVT, orientación este. Fuente: Elaboración de los autores.

Tomando valores de temperaturas promedio, se advierten magnitudes de $36.4^{\circ} \mathrm{C}$ en las máximas y $25.0^{\circ} \mathrm{C}$ en las mínimas, para el período 2019 , y valores de $38.5^{\circ} \mathrm{C}$ en las máximas y $24.3^{\circ} \mathrm{C}$ en las mínimas, para el período 2020 . Se verifican diferencias del orden de los $2.1^{\circ} \mathrm{C}$ respecto a las temperaturas máximas promedio, y diferencias de $0.7^{\circ} \mathrm{C}$ respecto a las temperaturas mínimas promedio entre ambos períodos considerados. Finalmente, tomando valores de temperaturas medias promedio, se encontraron magnitudes de $30.4^{\circ} \mathrm{C}$ y $30.9^{\circ} \mathrm{C}$ para los lapsos temporales de 2019 y 2020, respectivamente. Se evidencia que ambos períodos registran diferencias del orden de $0.5^{\circ} \mathrm{C}$ (Tabla 1).

Considerando la variable de amplitud térmica diaria, el rango de temperaturas oscila entre $9.8^{\circ} \mathrm{C}$ y $12.7^{\circ} \mathrm{C}$, para el primer período, y entre $11.2^{\circ} \mathrm{C}$ y $15.8^{\circ} \mathrm{C}$, para el segundo. En promedio, la amplitud térmica registra magnitudes de $11.4^{\circ} \mathrm{C}$ y $14.2^{\circ} \mathrm{C}$ para los períodos 2019 y 2020, respectivamente (Tabla 1).

\section{RESULTADOS Y DISCUSIÓN}

Prosiguiendo con el estudio, se seleccionaron tres variables -incidentes en el desempeño termo-energético de los casos a analizar- de la base de datos recopilada durante los días seleccionados del verano 2019 y 2020: temperatura superficial exterior, temperatura superficial interior y temperatura ambiente interior de espacios homólogos.
Dichas variables fueron analizadas en términos de la diferencia de temperatura entre ambos casos: caso con FVT y caso testigo. Ello se detalla a continuación.

\section{TEMPERATURA SUPERFICIAL EXTERIOR: CASO CON FVT VS. CASO TESTIGO}

A fin de reconocer potenciales impactos del uso de la vegetación en muros exteriores de mampostería con exposición al este, se compararon las temperaturas superficiales exteriores del caso con FVT (curva verde), el caso testigo (curva azul) y el comportamiento de la temperatura del aire exterior (curva roja) (Figura 7).

El caso con FVT presenta temperaturas superficiales exteriores menores respecto del caso testigo durante todas las horas diurnas. Las máximas reducciones de temperatura superficial exterior fueron de $26.5^{\circ} \mathrm{C}$ y $27.4^{\circ} \mathrm{C}$, para el primer y segundo período, respectivamente. Los picos de diferencia de temperatura se advierten, en ambos períodos, entre las 10:30 am y 10:45 am, en días de cielo claro (Figura 8). Lo anterior pone de manifiesto que la temperatura superficial exterior es fundamentalmente comandada por el fenómeno radiativo, teniendo en cuenta la orientación del muro -este-. Los resultados son superiores a las magnitudes encontradas por Vox, Blanco y Schettini (2018) y Hoelscher, Nehls, Jänicke y Wessolek (2016), las cuales oscilan entre $9.0^{\circ} \mathrm{C}$ y $15.5^{\circ} \mathrm{C}$ (FVT en orientación Norte y en clima del tipo Csa, mediterráneo templado cálido, FVT en orientación noroeste y clima del tipo Dfb, templado cálido, respectivamente). 


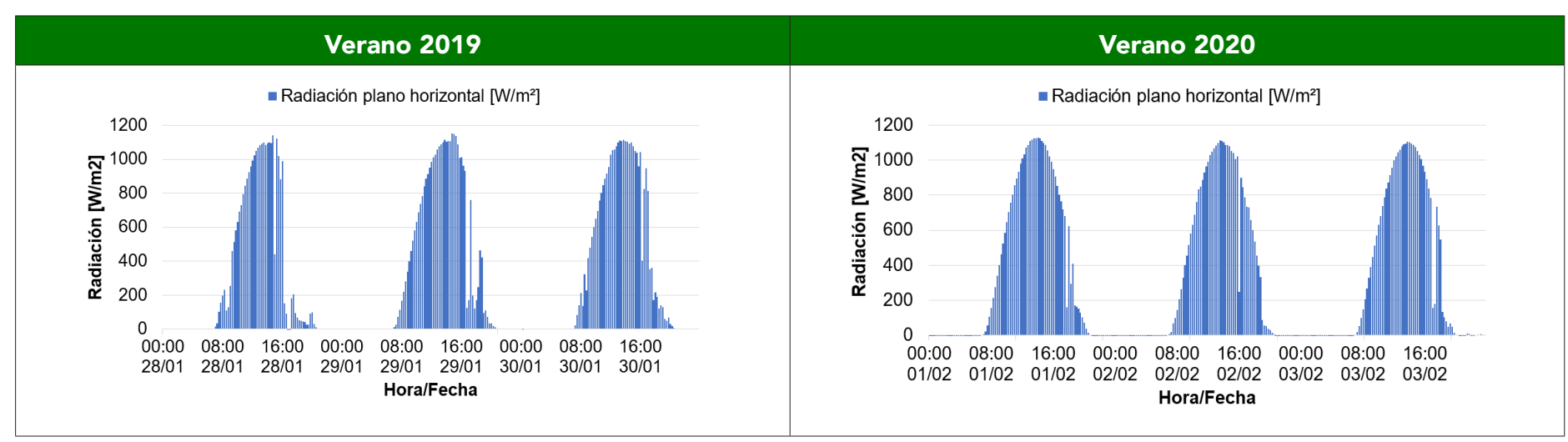

Figura 8. Irradiancia global en el plano horizontal para ambos períodos de estudio.Fuente: Elaboración de los autores.

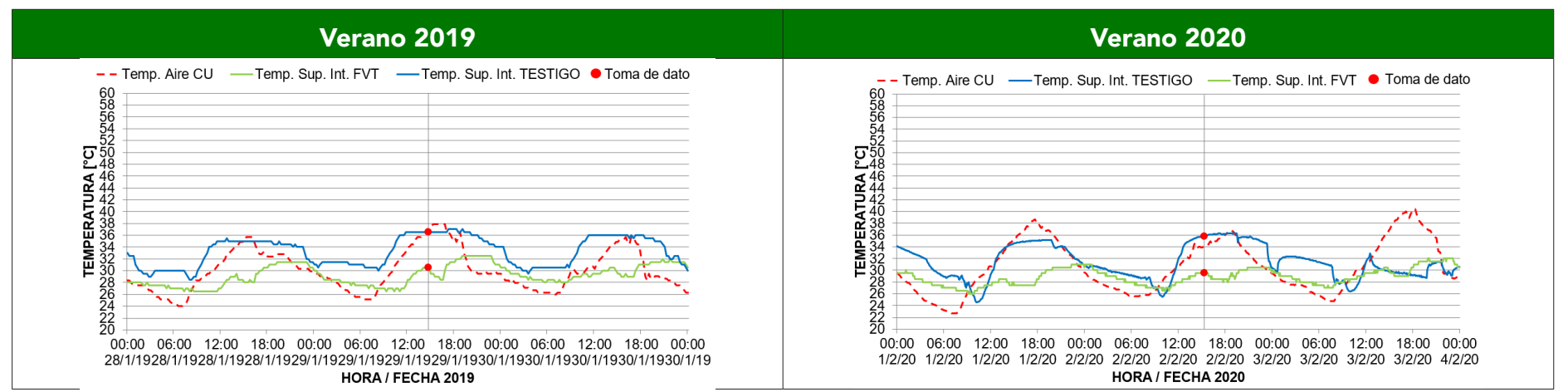

Figura 9. Temperatura superficial interior: caso testigo y caso con SEV, en orientación este. Fuente: Elaboración de los autores.

El análisis de las curvas de temperatura superficial del muro con FVT exhibe una amplitud térmica que oscila en torno los $6^{\circ} \mathrm{C}$ promedio en ambos períodos medidos. Por el contrario, la curva correspondiente al muro testigo registra una amplitud térmica de $24^{\circ} \mathrm{C}$ promedio en el primer período y de $31^{\circ} \mathrm{C}$ en el segundo período. Esto se debe a que el segundo período medido -2020- registró temperaturas más elevadas. El comportamiento del muro cubierto con vegetación muestra máximas y mínimas atenuadas que reflejan la capacidad conservativa del muro vegetado, comparable al comportamiento de un material aislante.

En el comportamiento de las curvas de la Figura 6, se aprecia que el efecto de enfriamiento producido por la FVT ocurre durante la totalidad de las horas diurnas en ambos períodos, donde las superficies exteriores de los muros están siempre más frías que las del caso testigo. Mientras que, por el contrario, el caso con FVT manifiesta temperaturas superficiales exteriores superiores respecto al caso testigo durante la noche del período 2020. Dicha magnitud registra un máximo de $4.1^{\circ} \mathrm{C}$ mayor en la superficie cubierta con vegetación, respecto del muro testigo. En consecuencia, la pared con FVT se encuentra más fresca durante el día y más caliente durante la noche, respecto al testigo en este período. Estos resultados se deben a la mayor amplitud térmica registrada en los días del verano 2020, donde el efecto aislante del muro con FVT puede percibirse durante la noche. No obstante, se debe tener en cuenta que la máxima magnitud de enfriamiento en el muro con FVT es 6.7 veces mayor que la máxima magnitud de calentamiento en relación al caso testigo, y se produce en horarios donde la temperatura ambiente exterior registra sus máximos valores.

Con el objetivo de verificar la respuesta de la temperatura superficial exterior en muros testigo y con FVT ante diferentes condiciones climáticas exteriores, y considerando que el verano 2020 presenta temperaturas máximas y promedio más elevadas que el verano 2019, se estableció una comparativa entre promedios de las máximas magnitudes diarias de esta variable. Se compararon entre sí los promedios de máximas registradas en el muro testigo y con FVT en los dos períodos. El muro testigo presenta, durante todo el período 2020, un aumento en el promedio de las máximas temperaturas superficiales exteriores de $7.3^{\circ} \mathrm{C}$ respecto de 2019. Mientras que el muro con FVT presenta, durante todo el período 2020, un aumento en el promedio de las máximas temperaturas superficiales exteriores de $4.5^{\circ} \mathrm{C}$ respecto de 2019 . Teniendo en cuenta que la temperatura del aire máxima promedio se incrementó entre ambos períodos en $2.2^{\circ} \mathrm{C}$, puede inferirse que mientras más riguroso es el verano, mayor es el impacto del FVT. 

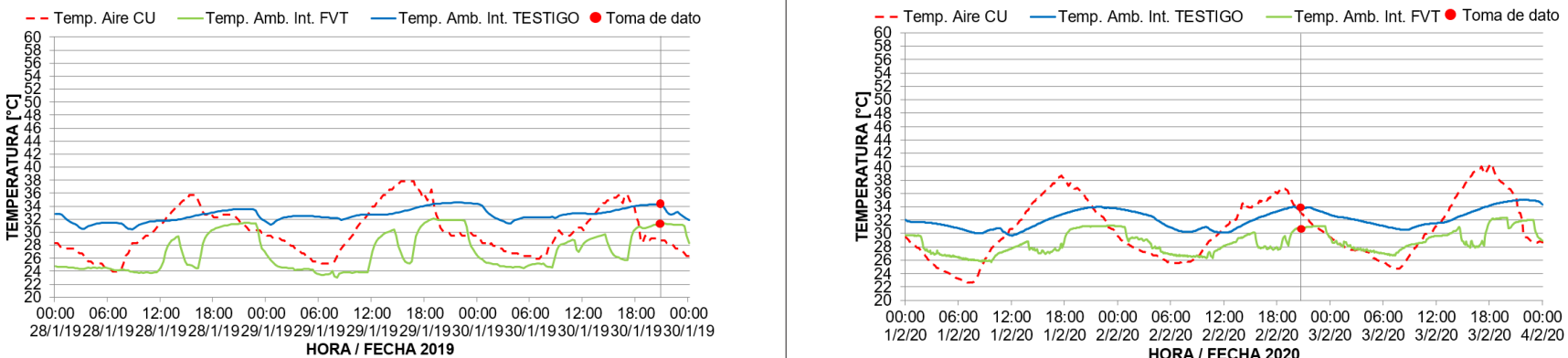

\section{TEMPERATURA SUPERFICIAL INTERIOR: CASO FVT VS. CASO TESTIGO}

Para determinar el impacto térmico del uso de la vegetación en el interior de las viviendas evaluadas, se midieron las temperaturas superficiales interiores. La Figura 9 ilustra el comportamiento de la temperatura superficial interior del muro con FVT (curva verde), del caso testigo (curva azul) y la temperatura del aire exterior (curva roja).

El caso con FVT muestra temperaturas superficiales interiores menores, respecto del caso testigo durante la totalidad de las horas diurnas, con reducciones máximas de $6.0^{\circ} \mathrm{C}$ y $6.5^{\circ} \mathrm{C}$ para los ciclos 2019 y 2020, respectivamente. Estas diferencias de temperatura se observan en ambos períodos entre las 14:45 y las 15:45 h: una y dos horas después del mediodía solar. Este retardo hallado, respecto del horario de máxima radiación, se puede interpretar como la consecuencia de la inercia térmica del paquete constructivo de los muros, lo cual es coherente con el comportamiento propio de materiales másicos de la envolvente. Ello pone de manifiesto que, al igual que la temperatura superficial exterior, la temperatura superficial interior está fuertemente condicionada por el fenómeno radiativo para esta orientación de muro -este-. Los resultados son superiores a las magnitudes encontradas por Hoelscher et al. (2016) y Susorova et al. (2013), los cuales oscilan entre $1.7^{\circ} \mathrm{C}$ y $2.0^{\circ} \mathrm{C}$ (FVT en orientación oeste y en clima del tipo Dfb templado cálido, FVT en orientación norte y en clima del tipo Dfa, continental verano caliente, respectivamente).

A diferencia del análisis de la temperatura superficial exterior, las magnitudes de enfriamiento en las superficies interiores de los muros, producidas por la presencia de vegetación, ocurren durante horarios diurnos y nocturnos en ambos períodos. Es decir, los efectos de enfriamiento de las FVT tienen impacto en el interior de las viviendas también durante horarios sin radiación solar. Ahora bien, es importante mencionar que las temperaturas superficiales interiores se ven afectadas por el uso del aire acondicionado en momentos que la temperatura ambiente exterior se aleja en mayor medida de la temperatura de confort. Esto es efecto del monitoreo de casos reales en condiciones de uso en la estación cálida. Es por ello que, en aras de atender al objetivo del trabajo, las diferencias de temperatura se tomaron en ausencia del uso del aire acondicionado.

\section{TEMPERATURA AMBIENTE INTERIOR: CASO FVT VS. CASO TESTIGO}

La Figura 10 grafica el comportamiento de la temperatura ambiente interior en dos espacios homólogos entre el caso con FVT (curva verde), el testigo (curva azul) y la temperatura del aire exterior (curva roja), para los dos períodos medidos.

El análisis de la temperatura ambiente interior permite identificar que la temperatura del caso con FVT es inferior a la del caso testigo, con máximas diferencias comprendidas entre $2.9^{\circ} \mathrm{C}$ y $3.1^{\circ} \mathrm{C}$ durante todas las horas del día en los dos ciclos de medición. Al igual que en lo observado en el análisis de las temperaturas superficiales, las diferencias son superiores a las encontradas por Kontoleon y Eumorfopoulou (2010), que fueron del orden de $0.5^{\circ} \mathrm{C}$ (FVT en orientación este y en clima del tipo tipo $\mathrm{Cfb}$, oceánico templado verano suave). Mientras que Hoelscher et al., (2016) y Perini, Ottelé, Fraaij, Haas y Raiteri (2011) no encontraron efectos del FVT sobre la temperatura del aire en espacios interiores.

La temperatura ambiente interior en la vivienda con FVT revela reducciones promedio respecto al caso testigo del orden de los $3^{\circ} \mathrm{C}$. Este resultado se puede interpretar como la magnitud del impacto del uso de las FVT en el calor sensible de una vivienda. Al igual que en el caso de la temperatura superficial interior, se observa en el comportamiento de la curva de temperatura interior, el uso del aire acondicionado. Es por ello que, también en este análisis, los datos fueron tomados en períodos sin acondicionamiento artificial de los espacios. 


\section{CONCLUSIÓN}

El trabajo expuesto evaluó el impacto de las FVT con orientación este, sobre las temperaturas superficiales exteriores e interiores, y la temperatura ambiente interior en viviendas de construcción de ladrillo cocido y estructura de hormigón armado. El estudio se desarrolló en el Área Metropolitana de Mendoza, en la estación verano, sobre un universo de análisis representativo, desde el punto de vista morfológico y tecnológico, de viviendas unifamiliares seriadas del área de estudio.

En términos cuantitativos, la investigación demuestra un potencial de disminución de las temperaturas superficiales exteriores en las envolventes de los edificios de hasta $27.4^{\circ} \mathrm{C}$ y de $6.5^{\circ} \mathrm{C}$, en las temperaturas superficiales interiores. Estos beneficios se deben al efecto de la estructura verde sobre el muro respecto a la exposición plena del muro testigo. Considerando los resultados hallados en la literatura internacional para otros climas y la misma tecnología constructiva -ladrillo y hormigón armado-, se advierte que la aplicación de FVT, como estrategia para mejorar el comportamiento térmico de espacios interiores, es más eficiente en climas áridos.

Además, las variaciones de temperatura entre los dos períodos medidos expresan que, frente a condiciones externas más rigurosas, es mayor el impacto del FVT en la atenuación de las temperaturas superficiales interiores y exteriores.

La comparación del comportamiento de las curvas de temperatura superficial de muro con FVT respecto al muro testigo, demuestra la capacidad de atenuación de las temperaturas máximas y mínimas resultantes del carácter conservativo de la FVT, que es comparable con el de un material aislante. Sin embargo, las FVT ofrecen ventajas adicionales respecto a la aplicación de materiales sintéticos para la conservación térmica ligadas a beneficios ecosistémicos y aportes a la salud de las personas. De esta conclusión, se desprende la necesidad de que, en futuras investigaciones, se tengan en cuenta los beneficios adicionales de las estrategias verdes para mensurar su eficiencia en relación a las estrategias de aislación tradicionalmente empleadas.

En cuanto a las temperaturas ambiente, se registraron reducciones del orden de $2.9^{\circ} \mathrm{C}$ y $3.1^{\circ} \mathrm{C}$, en los períodos 2020 y 2019, respectivamente, en la vivienda con FVT, respecto al caso testigo $\mathrm{y}$, asimismo, se constató que la magnitud promedio del impacto en el calor sensible de la vivienda es del orden de los $3^{\circ} \mathrm{C}$. Los valores de reducción de la temperatura interior alcanzados en este trabajo -al igual que los obtenidos respecto a la disminución de las temperaturas superficiales- son de mayor magnitud en comparación a los hallados en la literatura científica, en experiencias similares evaluadas en otros climas $\left(0.5^{\circ} \mathrm{C}\right)$. Estos resultados ponen en valor el uso de la estrategia para aminorar las temperaturas interiores y aproximar a las condiciones de confort a los espacios habitables dentro de los edificios en climas áridos.

Recapitulando, esta investigación representa un concreto aporte al desarrollo de conocimiento vinculado a la implementación de SEV en edificios emplazados en zonas climáticas del tipo árido desértico (BWk - Köppen-Geiger). En tal sentido, los resultados encontrados evidencian el potencial de una tecnología de enverdecimiento apropiada en relación con su eficiencia para disminuir las temperaturas y contribuir al ahorro energético resultante de una menor demanda de energía para el acondicionamiento térmico de los espacios interiores. Es decir, se presenta como una estrategia alternativa de aplicación simple en envolventes edilicias que tiende no sólo a garantizar la sustentabilidad del hábitat en contextos climáticos rigurosos, sino también a mejorar la calidad de vida del ambiente construido en el marco de un desarrollo sostenible.

En futuras etapas, se prevé incrementar el número de casos de estudio, evaluar el impacto asociado a otras tecnologías constructivas y al uso de diferentes especies para conformar FVT. El objetivo final es ampliar el alcance de esta investigación y generalizar resultados que contribuyan a potenciar el uso de la las FVT como herramienta de diseño para disminuir las temperaturas interiores en el ámbito construido y sus impactos asociados a escala edilicia, urbana y global.

\section{AGRADECIMIENTOS}

Los autores agradecen al Consejo Nacional de Investigación Científica y Tecnológica (CONICET) y a la Agencia Nacional de Promoción Científica y Tecnológica (ANPCYT) la financiación recibida para el desarrollo de esta investigación. Así también, a los propietarios de las viviendas Miguel Martí y Rodrigo Benítez que permitieron el monitoreo de los casos de estudio.

\section{REFERENCIAS BIBLIOGRÁFICAS}

Alexandri, E. y Jones, P. (2008). Temperature decreases in an urban canyon due to green walls and green roofs in diverse climates. Building and Environment, 43(4), 480-493. DOI: https:// doi.org/10.1016/j.buildenv.2006.10.055

Bustami, R. A., Belusko, M., Ward, J. y Beecham, S. (2018). Vertical greenery systems : A systematic review of research trends. Building and Environment, 146(August), 226-237. Recuperado de https://doi.org/10.1016/j.buildenv.2018.09.045

Coma, J., Pérez, G., De Gracia, A., Burés, S., Urrestarazu, M. y Cabeza, L. F. (2017). Vertical greenery systems for energy savings in buildings: A comparative study between green walls and green facades. Building and Environment, 111, 228-237. DOI: https:// doi.org/10.1016/j.buildenv.2016.11.014 
Contesse, M., Van Vliet, B. J. M. y Lenhart, J. (2018). Is urban agriculture urban green space? A comparison of policy arrangements for urban green space and urban agriculture in Santiago de Chile. Land Use Policy, 71(October), 566-577. DOI: https://doi.org/10.1016/j.landusepol.2017.11.006

Elsadek, M., Liu, B. y Lian, Z. (2019). Urban Forestry \& Urban Greening Green façades: Their contribution to stress recovery and well-being in high- density cities. Urban Forestry \& Urban Greening, 46(September). DOI: https://doi.org/10.1016/j. ufug.2019.126446

Gill, S. E., Handley, J. F., Ennos, A. R. y Pauleit, S. (2007). Adapting cities for climate change: The role of the green infrastructure. Built Environment, 33(1), 115-133. DOI: https:// doi.org/10.2148/benv.33.1.115

Haggag, M., Hassan, A. y Elmasry, S. (2014). Experimental study on reduced heat gain through green façades in a high heat load climate. Energy and Buildings, 82, 668-674. DOl: https://doi.org/10.1016/j.enbuild.2014.07.087

Hoelscher, M. T., Nehls, T., Jänicke, B. y Wessolek, G. (2016). Quantifying cooling effects of facade greening: Shading, transpiration and insulation. Energy and Buildings, 114, 283-290. Recuperado de https://doi.org/10.1016/j. enbuild.2015.06.047

Kontoleon, K. J. y Eumorfopoulou, E. A. (2010). The effect of the orientation and proportion of a plant-covered wall layer on the thermal performance of a building zone. Building and Environment, 45(5), 1287-1303. DOI: https://doi.org/10.1016/j. buildenv.2009.11.013

Marchi, M., Pulselli, R. M., Marchettini, N., Pulselli, F. M. y Bastianoni, S. (2015). Carbon dioxide sequestration model of a vertical greenery system. Ecological Modelling, 306, 46-56. DOI: https://doi.org/10.1016/j.ecolmodel.2014.08.013

OCED / IEA. (2017). Energy Technology Perspectives 2017. International Energy Agency (IEA) Publications. Recuperado de https://webstore.iea.org/download/ summary/237?fileName=English-ETP-2017-ES.pdf

Othman, A. R. y Sahidin, N. (2016). Vertical Greening Façade as Passive Approach in Sustainable Design. Procedia - Social and Behavioral Sciences, 222, 845-854. DOI: https://doi. org/10.1016/j.sbspro.2016.05.185

Pérez, G., Coma, J., Sol, S. y Cabeza, L. F. (2017). Green facade for energy savings in buildings: The influence of leaf area index and facade orientation on the shadow effect. Applied Energy, 187, 424-437. DOI: https://doi.org/10.1016/j. apenergy.2016.11.055

Perini, K., Ottelé, M., Fraaij, A. L. A., Haas, E. M. y Raiteri, R. (2011). Vertical greening systems and the effect on air flow and temperature on the building envelope. Building and Environment, 46(11), 2287-2294. DOI: https://doi. org/10.1016/j.buildenv.2011.05.009

Salas-Esparza, M. G. y Herrera-Sosa, L. C. (2017). La vegetación como sistema de control para las Islas de Calor Urbano en Ciudad Juárez, Chihuahua. Hábitat Sustentable, 7(1), 14-23. Recuperado de http://revistas.ubiobio.cl/index.php/RHS/ article/view/2737
Suklje, T., Saso, M. y Arkar, C. (2016). On detailed thermal response modeling of vertical greenery systems as cooling measure for buildings and cities in summer conditions. Energy, 115, 10551068. DOI: https://doi.org/10.1016/j.energy.2016.08.095

Susorova, I., Angulo, M., Bahrami, P. y Stephens, B. (2013). A model of vegetated exterior facades for evaluation of wall thermal performance. Building and Environment, 67, 1-13. DOI: https:// doi.org/10.1016/j.buildenv.2013.04.027

Vox, G., Blanco, I. y Schettini, E. (2018). Green façades to control wall surface temperature in buildings. Building and Environment, 129(September 2017), 154-166. DOI: https://doi.org/10.1016/j. buildenv.2017.12.002

Wong, I. y Baldwin, A. N. (2016). Investigating the potential of applying vertical green walls to high-rise residential buildings for energy-saving in sub-tropical region. Building and Environment, 97, 34-39. DOI: https://doi.org/10.1016/j.buildenv.2015.11.028

Wong, N. H., Kwang Tan, A. Y., Tan, P. Y., Chiang, K. y Wong, N. C. (2010). Acoustics evaluation of vertical greenery systems for building walls. Building and Environment, 45(2), 411-420. DOl: https://doi.org/10.1016/j.buildenv.2009.06.017 